\title{
Received Signal Strength Indication Modeling in Indoor Wireless Sensor Networks
}

\author{
Edson Taira Procopio, Alexandre de Assis Mota, \\ Lia Toledo Moreira Mota and Luiz Rodolfo Barreto da Silva \\ Department of CEATEC, Faculty of Electrical Engineering, \\ Pontifical Catholic University of Campinas-São Paulo, CEP 13086-900, Brazil
}

Received 2013-05-14, Revised 2013-07-29; Accepted 2013-08-06

\begin{abstract}
This study aims to identify mathematical models that represent the relation between Received Signal Strength Indication (RSSI) and objects in an indoor Wireless Sensor Network (WSN). Using the Least Squares Method, four linear models have been identified: The first one relates uplink RSSI and objects; the second one relates downlink RSSI and objects; the third one relates uplink RSSI and obstacles and the fourth one relates downlink RSSI and obstacles. The obtained results, characterized by small residual values, attest the validation of all four models.
\end{abstract}

Keywords: Mathematical Modeling, Wireless Sensor Networks, Least Square Method

\section{INTRODUCTION}

Nowadays, Wireless Sensor Networks (WSNs) are widely applied in residential, commercial and industrial monitoring (Doherty et al., 2001; HevinRajesh and Paramasivan, 2012). They are employed, for instance, in monitoring light, temperature and energy consumption in the built environment (Camilo et al., 2006) and in several applications of the Smart Grid concept, WSNs can also play an important role.

An WSN is an ad-hoc network composed by nodes with the capacity of collecting, processing and transmitting, in an autonomous way, data related to the area they are placed (Qian and Zhang, 2009). These networks are formed by sensor nodes, that are responsible for collecting information and for sending these data to the base node, which is connected to a computer. Figure 1 illustrates an ordinary configuration of a WSN.

There is a great concern about the reliability of the information collected by the sensors and transmitted to the base and, consequently, there is a need for knowing characteristics related to the sensor-base communication. One way of studying the communication between a sensor node and the base node is by analysing the Received Signal Strenght Indication (RSSI), since it has been proven that small values of RSSI have a negative impact on the WSN reliability (Camilo et al., 2006).

The RSSI indicates the intensity of the received signal and can be measured considering the signal transmitted from the base to the sensor node (uplink) or from the sensor node to the base (downlink). Since the RSSI varies according to indoor and outdoor physical aspects, it is of fundamental importance to determine its behavior as a function of indoor and outdoor characteristics.

In this context, the objective of this work is to define a mathematical model to represent the RSSI behavior between two nodes (sensor and base) of an indoor WSN, as a function of elements that can influence this behavior, thus taking into account the influences that objects or obstacles can offer to data transmission.

This study is organized as follows. In the following, materials and methods used for determining the mathematical model are described. Next, the obtained results are shown and, finally, the conclusions of the work are presented.

Corresponding Author: Edson Taira Procopio, Department of CEATEC, Faculty of Electrical Engineering, Pontifical Catholic University of Campinas-São Paulo, CEP 13086-900, Brazil 


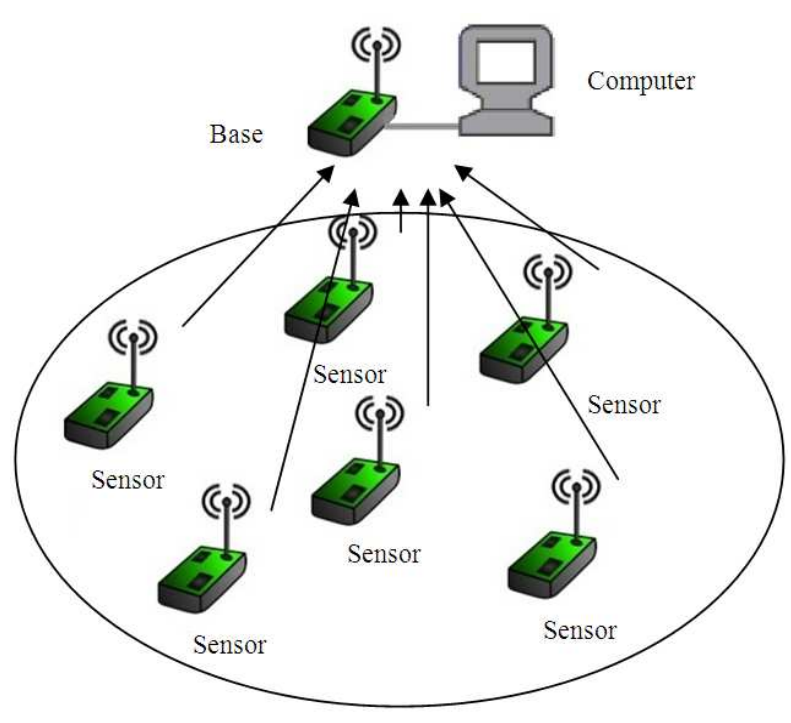

Fig. 1. Ordinary configuration of an WSN

\section{MATERIALS AND METHODS}

In order to study the indoor RSSI behavior, it was necessary, first of all, to establish communication between a sensor node and a base node, creating a WSN operating according to IEEE802.15.4 standards. Then, aiming to study the impact of indoor characteristics on this communication signal, the uplink RSSI and the downlink RSSI were analised, considering the sensor node placed at different positions.

\subsection{Wireless Sensor Network}

The sensor node was composed by an RFBee sensor module and was placed at an interest point, connected only to a battery. The base node was composed by connecting an RFBee sensor module to a base module and, then, by connecting these modules, using an Universal Serial Bus (USB) cable, to a computer, which was responsible for receiving the information sent from the base and also for providing the power supply for the base node.

The RFBee hardware platform is free and compatible with the Arduino platform, which has been widely used in electronic projects using hardware with embedded software, because of owning a simple programming language. Figure 2 shows an RFBee sensor module and Fig. 3 shows a base module, where the sensor module is attached and connected directly to a computer unit.

The RFBee modules have an ATmega168 microcontroller, from Atmel, which can be programmed according to the required application and also owns the
Integrated Circuit (IC) CC1101 from Texas Instruments.. The IC is responsible for the CC1101 radio transmission frequency. The sensitivity, operating at a frequency of $915 \mathrm{MHz}$ is-112 dBm, according to technical specifications (ATMEL, 2012).

The configuration parameters for the signals emitted from the nodes must be defined at the computational programs saved in the RFBee modules. In this study, these parameters were defined, for the sensor and for the base nodes, as described in Table 1.

\subsection{RSSI Modeling}

In order to determine the mathematical model that represents the RSSI behavior as a function of indoor elements (objects and obstacles), uplink and downlink RSSI measurements were collected for the WSN previously described.

As the model would be based on data collected from the System Under Analysis (WSN), the modelling approach chosen to determine the mathematical model was the black-box modelling technique (Mota, 2005; Yano et al., 2013). In this approach the model is determined based on input and output system data. In this study, indoor obstacles and objects were the input data and the uplink and downlink RSSI were the output data.

The model was determined using the System Identification technique, which comprehends five steps: data collectinh, model type chosen, model structure chosen, parameter estimation and model validation

\subsection{Data Collection}

To perform data collection, tests were carried out considering the communication between the base node and the sensor node, as described before. In each test, uplink and downlink RSSI were collected and indoor objects and obstacles were identified. In this work, objects are defined as indoor physical elements that are not placed in the line of sight between the sensor and the base nodes, while obstacles are considered as objects that are placed in the line of sight between these nodes.

The tests were performed at three different places: classrooms, an electronic laboratory and a transmission medium laboratory, all located at Pontifical Catholic University of Campinas (PUC-Campinas). These places were chosen because of their different indoor characteristics, with different objects and obstacles. In all tests, the WSN nodes were placed at the same height, at $0,8 \mathrm{~m}$ from the floor:

Test 1: The first test was carried out on four classrooms of PUC-Campinas. Figure 4 illustrates these four classrooms (A, B, C and D). In Test 1, input and output data were collected for 10 different 
situations. In this figure, B1 represents the base node which remains fixed for all situations. The sensor node was placed at different locations for each situation and these locations are represented, in Fig. 4, by numbers from 1 to 10 .

Test 2: The second test was carried out on four rooms of the Electronic Laboratory of PUC-Campinas. Fig. 5 illustrates these four rooms (A, B, C and D). In Test 2, input and output data were collected for 26 different situations. In this figure, B1 and B2 represent the base node. This node was firstly fixed at position B1 for the sensor node placed at positions from 1 to 13 . Then, the base node was fixed at position B2 for the sensor node placed at positions from 14 to 26 .

Test 3: The third test was carried out on four rooms of the Transmission Medium Laboratory of PUCCampinas. Figure 6 illustrates these four rooms (A, B, C and D). In Test 3, input and output data were collected for 22 different situations. In this figure, B1 and B2 represent the base node.

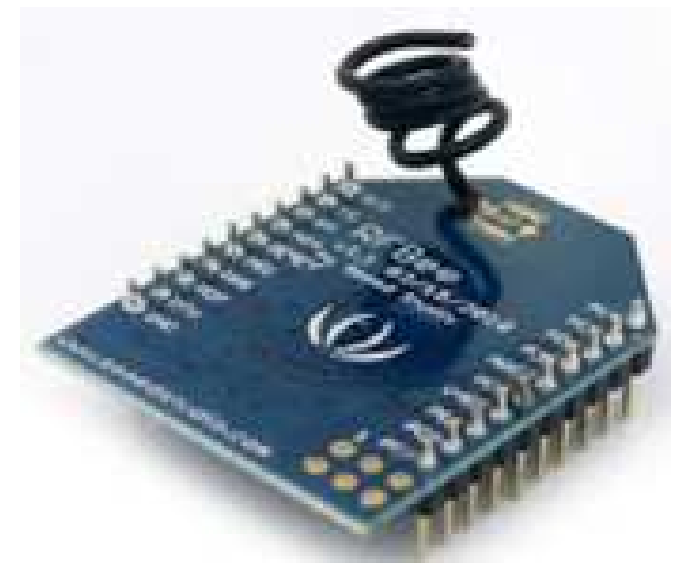

Fig. 2. RFBee sensor module (10)

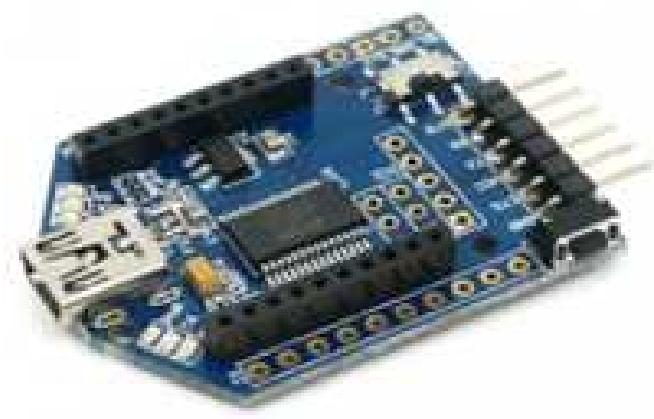

Fig. 3. RFBee base module (11)
This node was firstly fixed at position B1 for the sensor node laced at positions from 1 to 11 . Then, the base node was fixed at position B2 for the sensor node placed at positions from 12 to 22 .

\subsection{Model Type and Model Structure}

After collected, the input and output data were plotted together in order to visually identify a relarion between the uplink and downlink RSSI (output data) and the objects and obstacles presented in the rooms (input data).

Based on this plot, the type and the structure of the model could be determined as being linear, since the uplink and the downlink RSSI decrease with the increase of the number of objects and obstacles, acccording to a straight line. Even with the variety of objects involved, with different impacts on communication, the values contained in the database tend to exhibit this behavior.

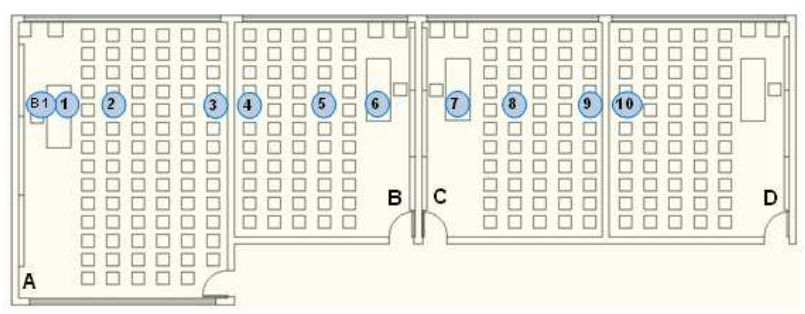

Fig. 4. Classrooms where Test 1 was carried out

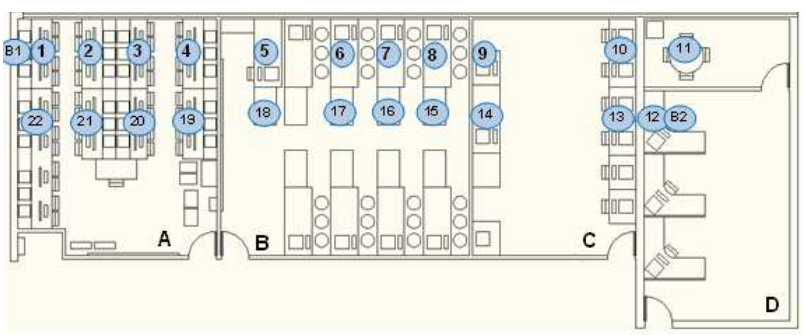

Fig. 5. Rooms where Test 2 was carried out

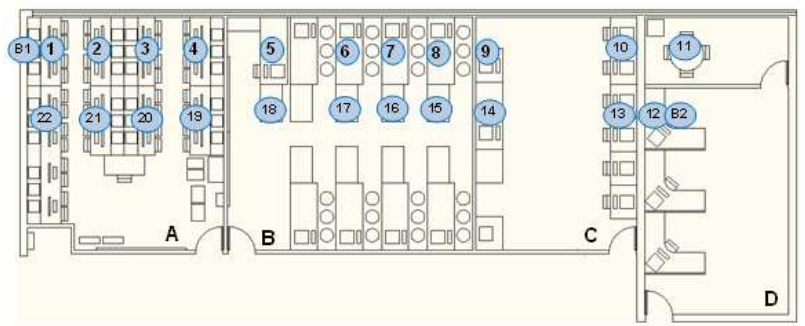

Fig. 6. Rooms where Test 3 was carried out 


\subsection{Parameters Estimation}

The results of RSSI Modeling lead to assume the hypothesis that the system under study is a discrete linear system, with multiple inputs. This system can be described according to Equation 1:

$$
\mathrm{Y}=(\mathrm{X})^{\mathrm{T}} \cdot \theta
$$

Where:

$\mathrm{Y}=$ The output vector of the system

$\mathrm{X}=$ The input vector/matrix of the system

$\theta=$ The model parameters vector

In this study, the $\mathrm{Y}$ vector is composed by the RSSI measurements and the $\mathrm{X}$ matrix is composed by the indoor objects and obstacles.

The parameter vector of this model was estimated applying the Least Squares Method, according to Equation 2:

$$
\theta_{\text {est }}=\left[X^{\mathrm{T}} \cdot \mathrm{X}\right]^{-1} \cdot \mathrm{X}^{\mathrm{T}} \cdot \mathrm{Y}
$$

\subsection{Model Validation}

In order to validate the obtained model, a residual analysis was carried out. This means that for each estimated model, the residual vector was calculated as described in Equation (3):

$$
\mathrm{E}=\mathrm{Y}-\mathrm{X} \cdot \theta_{\text {est }}
$$

where, $\mathrm{Y}$ is the output vector, composed by RSSI measurements; $\mathrm{X}$ is the input matrix, composed by indoor objects and obstacles and $\theta_{\text {est }}$ is the parameters vector estimated according to Equation (2).

Then, for each estimated model, the Mean Square Residual (MSR) was calculated, according to Equation (4):

$$
\operatorname{MSR}=\frac{1}{n} \sum_{i=1}^{n}\left(E_{i}\right)^{2}=\frac{1}{n} \sum_{i=1}^{n}\left(Y_{i}-X_{i} \cdot \theta_{\text {est }}\right)^{2}
$$

where, $\mathrm{n}$ is the total number of samples.

\section{RESULTS}

Tests were performed for 58 different conditions (Tests 1, 2 and 3 previously described) and each test lasted for $3 \mathrm{~min}$.

The tests resulted in the database with input data, containing information about indoor objects and obstacles and output data, which were the RSSI (downlink and uplink) values. This database was stored on the laptop that was connected to the base node.
The uplimk and downlink RSSI value were divided into two parts. The first part contained 30 different conditions (considering Tests 1, 2 and 3) and input and output data were used to estimate the mathematical model.

The second part contained 28 different conditions (also onsidering Tests 1, 2 and 3) and input and output data were used to validate the obtained model.

\subsection{Indoor Objects}

The input data was collected by identifying the types and quantities of indoor objects and obstacle. The indoor objects that were quantified in tests are shown in Table 2.

The quantification of the objects described in Table 2, regarding the 30 tests used for model identification is presented in Table 3. The first line of the table indicates the identification number of each object corresponding to Table 2 and the first column is the number corresponding to the test performed.

\subsection{Indoor Obstacles}

The other way to enter the information in the database was to quantify the number of obstacles between the sensor node and the base node. These obstacles are shown in Table 4. The quantification of the obstacles described in Table 4, regarding the 30 tests used for model identification is presented in Table $\mathbf{5}$.

\subsection{Identified Model for the Relation between Uplink RSSI and Objects}

Using the System Identification technique, described before, it was possible to identify a mathematical model relating the presence of indoor objects to the uplink RSSI of an indoor WSN. This model is described by Equation (1). For this case the obtained parameters vector is described by Equation (5) and the mean square residual (MSR) is described by Equation (6).

\subsection{Identified Model for the Relation between Downlink RSSI and Objects}

Using the System Identification technique, it was also possible to identify a mathematical model relating the presence of indoor objects to the downlink RSSI of an indoor WSN. This model is described by Equation (1). For this case the obtained parameters vector is described by Equation (7). The mean square residual (MSR) is described by Equation (8).

\subsection{Identified Model for the Relation between Uplink RSSI and Obstacles}

It was also possible to identify a mathematical model relating the presence of indoor obstacles to the 
uplink RSSI of an indoor WSN. This model is described by Equation (1). For this case the obtained parameters vector is described by Equation (9). The mean square residual (MSR) is described by Equation (10).

\subsection{Identified Model for the Relation between Downlink RSSI and Obstacles}

Finally, it was possible to identify a mathematical model relating the presence of indoor obstacles to the downlink RSSI of an indoor WSN. This model is described by Equation (1). For this case the obtained parameters vector is described by Equation (11) and the Mean Square Residual (MSR) is described by Equation (12):

$\theta$ est $=1.0 \mathrm{e}-003 *\left[\begin{array}{c}-0.0126 \\ 0.3061 \\ -0.0655 \\ -0.4023 \\ -0.0664 \\ 0.0051 \\ 0.7296 \\ -0.0092 \\ -0.0011 \\ -0.0174 \\ 0.0279 \\ 0.0655 \\ -0.1221 \\ 0.0394 \\ -0.10696 \\ 0.2071 \\ 0.2640 \\ -0.0447 \\ -0.0180 \\ -0.0314 \\ 0.0159 \\ -0.0040 \\ 0.0169 \\ -0.1486 \\ 0.1035\end{array}\right]$

$\operatorname{MSR}=5.4077 \mathrm{e}-007 \mathrm{~mW}^{2}$

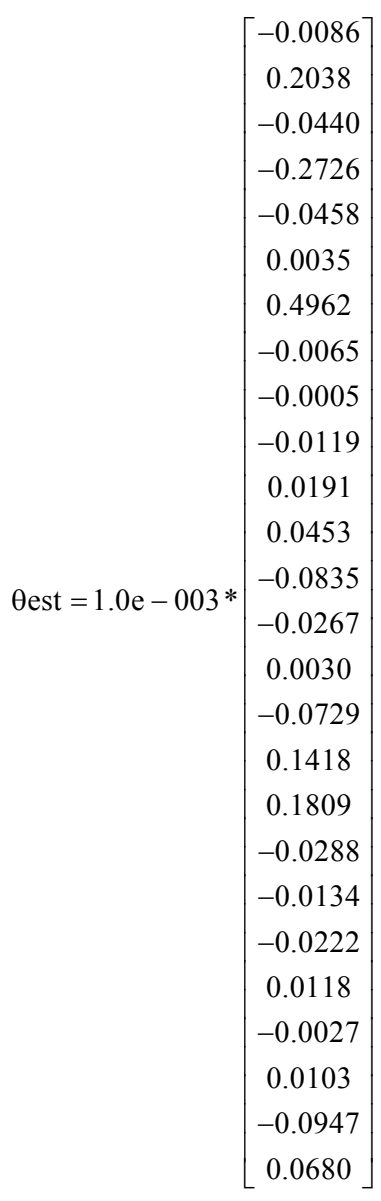

(7)

$\operatorname{MSR}=2.4991 \mathrm{e}-007 \mathrm{~mW}^{2}$

(8)

Table 1. Sensor and base nodes parameters

\begin{tabular}{ll}
\hline Parameter & Value \\
\hline Transmitted signal power & $5 \mathrm{~mW}$ \\
Transmission channel & 8 \\
Signal modulation & GFSK \\
Operating frequency & $915 \mathrm{MHz}$ \\
\hline
\end{tabular}

Table 2. Indoor objects types

1 - Desktop computer
2 - Laptop
3 - Switch
4 - Printer
5 - Blackboard
6 - Chair
7 - Fountain
8 - Measuring Equipment
9 - Seat
10 - Big table
11 - Small table
12 - Stand
13 - Big window

14 - Small window 15 - Luminaire 16 - Ventilator 17 - Big rack 18 - Small Rack 19 - Wi - fi Antenna 20 - Switchboard 21 - Large metal cabinet 22 - Small metal cabinet 23 - Small wood cabinet 24 - Door 25 - Air conditioner 26 - Speaker 
Edson Taira Procopio et al. / American Journal of Applied Sciences 10 (9): 1043-1049, 2013

Table 3. Indoor objects matrix

\begin{tabular}{|c|c|c|c|c|c|c|c|c|c|c|c|c|c|c|c|c|c|c|c|c|c|c|c|c|c|c|}
\hline & 1 & 2 & 3 & 4 & 5 & 6 & 7 & 8 & 9 & 10 & 11 & 12 & 13 & 14 & 15 & 16 & 17 & 18 & 19 & 20 & 21 & 22 & 23 & 24 & 25 & 26 \\
\hline 1 & 21 & 1 & 11 & 0 & 2 & 37 & 0 & 0 & 0 & 9 & 1 & 0 & 1 & 1 & 9 & 0 & 2 & 2 & 0 & 0 & 1 & 0 & 1 & 2 & 0 & 0 \\
\hline 2 & 21 & 1 & 11 & 0 & 2 & 37 & 0 & 0 & 0 & & 1 & 0 & 1 & 1 & 9 & 0 & 2 & 2 & 0 & 0 & 1 & 0 & 1 & 2 & 0 & 0 \\
\hline & 30 & 1 & 11 & 0 & 4 & 38 & 0 & 0 & 32 & 18 & 9 & 0 & 2 & 10 & 21 & 0 & 2 & 2 & 0 & 0 & 2 & 0 & 2 & 4 & 0 & 0 \\
\hline 4 & 30 & 1 & 11 & 0 & 4 & 38 & 0 & 0 & 32 & 18 & 9 & 0 & 2 & 10 & 21 & 0 & 2 & 2 & 0 & 0 & 2 & 0 & 2 & 4 & 0 & \\
\hline & 38 & 1 & 11 & 1 & 4 & 55 & 0 & 0 & 32 & 18 & 19 & & 3 & 12 & 27 & 0 & 2 & 2 & 0 & 0 & 2 & 0 & 3 & 5 & 0 & 0 \\
\hline 6 & 41 & 1 & 11 & 1 & 4 & 62 & 0 & 0 & 32 & 18 & 23 & 0 & 4 & 14 & 29 & 0 & 3 & 2 & 0 & 1 & 2 & 0 & 4 & 5 & 0 & 0 \\
\hline & 11 & 0 & 0 & 1 & 0 & 24 & 0 & 0 & 0 & 0 & 14 & 0 & 2 & 4 & 8 & 0 & 1 & 0 & 0 & 1 & 0 & 0 & 2 & 1 & 0 & 0 \\
\hline 8 & 20 & 0 & 0 & 1 & 2 & 25 & 0 & 0 & 32 & 9 & 22 & 0 & 3 & 13 & 20 & 0 & 1 & 0 & 0 & 1 & 1 & 0 & 3 & 3 & 0 & \\
\hline & 20 & 0 & 0 & 1 & 2 & 25 & 0 & 0 & 32 & 9 & 22 & 0 & 3 & 13 & 20 & 0 & 1 & 0 & 0 & 1 & 1 & 0 & 3 & 3 & 0 & 0 \\
\hline 10 & 41 & 1 & 11 & 1 & 3 & 62 & 0 & 0 & 32 & 18 & 23 & & 6 & 13 & 29 & 0 & 3 & 2 & 0 & 1 & 2 & 0 & 4 & & 0 & 0 \\
\hline 11 & 41 & 1 & 11 & 1 & 3 & 62 & 0 & 0 & 32 & 18 & 23 & 0 & 6 & 13 & 29 & 0 & 3 & 2 & 0 & 1 & 2 & 0 & 4 & & 0 & 0 \\
\hline 12 & 18 & 1 & 0 & 0 & 1 & 42 & 0 & 0 & 1 & 9 & 2 & 0 & 0 & 12 & 10 & 0 & 0 & 0 & 0 & 1 & 0 & 0 & 1 & 2 & 0 & 0 \\
\hline & 18 & 1 & 0 & 0 & 1 & 42 & 0 & 0 & 1 & 9 & 2 & 0 & 0 & 12 & 10 & 0 & 0 & 0 & 0 & 1 & 0 & 0 & 1 & 2 & 0 & 0 \\
\hline & 23 & 1 & 1 & 1 & 1 & $4 \varepsilon$ & 1 & 6 & 1 & 9 & 5 & 2 & 0 & 27 & 16 & 0 & 0 & & 0 & 1 & 3 & 5 & 2 & 3 & 0 & 0 \\
\hline & 23 & 1 & 1 & 1 & 1 & 48 & 1 & 6 & 1 & 9 & 5 & 2 & 0 & 27 & 16 & 0 & 0 & 0 & 0 & 1 & 3 & 5 & 2 & & 0 & 0 \\
\hline 16 & 23 & 1 & 1 & 1 & 1 & 48 & 1 & 6 & 1 & 9 & 5 & 2 & 0 & 27 & 16 & 0 & 0 & 0 & 0 & 1 & 3 & 5 & 2 & 3 & 0 & 0 \\
\hline & 33 & 1 & 1 & 1 & 2 & 68 & 1 & 6 & 1 & 9 & 15 & 2 & 0 & 42 & 19 & 0 & 0 & 0 & 0 & 1 & 3 & 5 & 3 & 4 & 0 & 0 \\
\hline & 33 & 1 & 1 & 1 & 3 & 104 & 1 & 42 & 1 & 9 & 16 & 8 & 0 & 5 & 3 & 0 & & & 0 & 2 & 3 & 5 & 4 & 6 & 1 & 0 \\
\hline & 0 & 0 & 0 & 0 & 1 & 36 & 0 & 36 & 0 & 0 & & & & 1 & 1 & & & & 0 & 1 & 0 & 0 & & & 1 & 0 \\
\hline 20 & 10 & 0 & 0 & 0 & 2 & 56 & 0 & 36 & 0 & 0 & 11 & & 0 & 2 & 1 & 0 & & & 0 & 1 & 0 & 0 & 2 & & 1 & 0 \\
\hline & 15 & 0 & 1 & 1 & 2 & 62 & 1 & 42 & 0 & 0 & 14 & 8 & 0 & 41 & 2 & 0 & 0 & 0 & 0 & 1 & 3 & 5 & 3 & 4 & 1 & 0 \\
\hline & 15 & 0 & 1 & 1 & 2 & 62 & & 42 & 0 & 0 & 14 & 8 & 0 & 4 & 2 & 0 & ( & & 0 & 1 & 3 & 5 & 3 & 4 & 1 & 0 \\
\hline & 33 & 1 & 1 & 1 & 3 & 104 & & 42 & 1 & 9 & 16 & 8 & 0 & 5 & 3 & 0 & & & 0 & 2 & 3 & 5 & 4 & 6 & 1 & 0 \\
\hline & 33 & 1 & 1 & 1 & 3 & 10 & 1 & 42 & 1 & 9 & 16 & & & 5 & 3 & & & & 0 & 2 & 3 & 5 & 4 & & 1 & 0 \\
\hline & 0 & 1 & 0 & 0 & 3 & 10 & 0 & 0 & 0 & 0 & & & & 2 & 14 & & & & 0 & 0 & 0 & 1 & 1 & & 1 & 2 \\
\hline & 0 & 1 & 0 & 0 & 3 & 10 & 0 & 0 & 0 & 0 & 1 & 0 & 0 & 2 & 1 & 6 & & 1 & 0 & 0 & 0 & 1 & 1 & 0 & 1 & 2 \\
\hline & 0 & 1 & 0 & 0 & 6 & 18 & & 0 & 0 & 0 & 2 & 0 & 0 & 28 & 2 & 12 & & 2 & 1 & 0 & 0 & 1 & 2 & 0 & 2 & 4 \\
\hline & 0 & 1 & 0 & 0 & 9 & 255 & () & 0 & 0 & 0 & 3 & 0 & 0 & 3 & 3 & 1 & & & 1 & 0 & 0 & 1 & 3 & 0 & 3 & 6 \\
\hline & 0 & 1 & 0 & 0 & 9 & 255 & 0 & 0 & 0 & 0 & 3 & ( & 0 & 35 & 37 & 18 & & & 1 & 0 & 0 & 1 & 3 & & 3 & 6 \\
\hline 30 & 0 & 1 & 0 & 0 & 12 & 329 & 0 & 0 & 0 & 0 & 4 & 0 & 0 & 42 & 48 & 24 & 0 & 4 & 1 & 0 & 0 & 1 & 4 & 0 & 4 & 8 \\
\hline
\end{tabular}

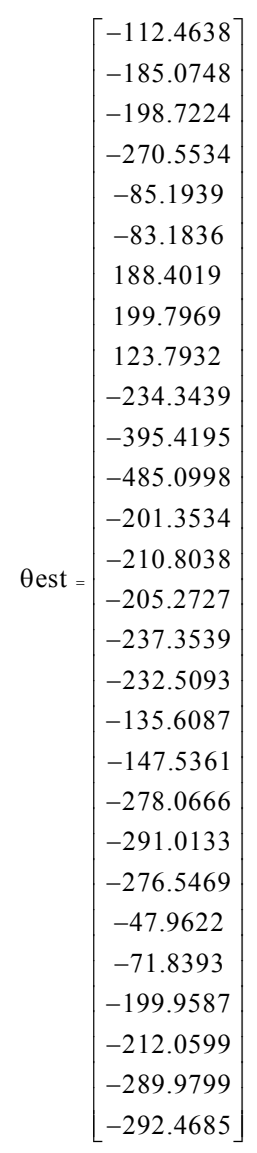

Table 4. Indoor obstacles

\begin{tabular}{ll}
\hline 1 & Chair \\
2 & Plywood Wood \\
3 & Metal conduit \\
4 & Blackboard \\
5 & Wall \\
6 & Glass/Window \\
7 & Wood cabinet \\
\hline
\end{tabular}

Table 5. Indoor obstacles matrix

\begin{tabular}{llllllll}
\hline & 1 & 2 & 3 & 4 & 5 & 6 & 7 \\
\hline 1 & 0 & 0 & 0 & 0 & 0 & 0 & 0 \\
2 & 2 & 1 & 2 & 0 & 0 & 0 & 0 \\
3 & 4 & 2 & 3 & 1 & 0 & 0 & 0 \\
4 & 4 & 2 & 3 & 1 & 0 & 0 & 0 \\
5 & 4 & 3 & 4 & 1 & 0 & 0 & 0 \\
6 & 6 & 3 & 6 & 1 & 1 & 0 & 0 \\
7 & 0 & 0 & 2 & 0 & 1 & 0 & 0 \\
8 & 2 & 1 & 3 & 0 & 1 & 0 & 0 \\
9 & 2 & 1 & 3 & 0 & 1 & 0 & 0 \\
10 & 2 & 2 & 4 & 1 & 1 & 0 & 0 \\
11 & 4 & 3 & 6 & 1 & 1 & 0 & 0 \\
12 & 0 & 0 & 0 & 0 & 0 & 0 & 0 \\
13 & 0 & 1 & 2 & 0 & 0 & 0 & 0 \\
14 & 0 & 1 & 2 & 0 & 0 & 1 & 0 \\
15 & 0 & 2 & 3 & 0 & 0 & 1 & 1 \\
16 & 0 & 3 & 5 & 0 & 0 & 1 & 1 \\
17 & 0 & 4 & 6 & 0 & 0 & 2 & 2 \\
18 & 0 & 5 & 8 & 0 & 0 & 3 & 2 \\
19 & 0 & 0 & 0 & 0 & 0 & 0 & 0 \\
20 & 0 & 1 & 2 & 0 & 0 & 1 & 0 \\
21 & 0 & 2 & 3 & 0 & 0 & 2 & 1 \\
22 & 0 & 2 & 3 & 0 & 0 & 2 & 0 \\
23 & 0 & 4 & 6 & 0 & 0 & 3 & 2 \\
24 & 0 & 5 & 8 & 0 & 0 & 3 & 2 \\
25 & 0 & 0 & 0 & 0 & 0 & 0 & 0 \\
26 & 5 & 0 & 0 & 0 & 0 & 0 & 0 \\
27 & 10 & 0 & 0 & 0 & 1 & 0 & 0 \\
28 & 14 & 0 & 0 & 0 & 2 & 0 & 0 \\
29 & 19 & 0 & 0 & 0 & 2 & 0 & 0 \\
30 & 20 & 0 & 0 & 0 & 3 & 0 & 0 \\
\hline
\end{tabular}


$\operatorname{MSR}=1.4023 \mathrm{e}-009 \mathrm{~mW}^{2}$

$\theta$ est $=1.0 \mathrm{e}-007 *\left[\begin{array}{c}0.0402 \\ 0.0196 \\ 0.1918 \\ -0.8106 \\ -0.3366 \\ -0.1627 \\ -0.4737\end{array}\right]$

$\mathrm{MSR}=5.5862 \mathrm{e}-010 \mathrm{~mW}^{2}$

\section{DISCUSSION}

From the obtained results, one can conclude that the models identified using the Least Squares Method, are able to represent the relation between RSSI (downlink or uplink) and objects (or obstacles) in an indoor WSN.

\section{CONCLUSION}

The small MSR values found for all four identified models show that these models are adequate to represent the mentioned relation, considering the communication between one sensor node and one base node. This means that the adopted hypothesis of a linear relation between the mentioned variables is valid for this indoor WSN. This realation can be considered as a starting point to future works that will aim to study more complex WSNs.

\section{REFERENCES}

ATMEL, 2012. ATMEGA168-8-bit Microcontroller with $8 \mathrm{~K}$ Bytes In-System Programmable Flash. Component Datasheet.
Camilo, T., A. Rodrigues, J.S. Silva and F. Boavida, 2006. Lessons learned from a real wireless sensor network deployment. Proceedings of the IFIP Networking Conference on Performance Control in Wireless Sensor Networks, (PCWSN' 06), pp: 80-88.

Doherty, L., K.S.J. Pister and L.E. Ghaoui, 2001. Convex position estimation in wireless sensor networks. Proceedings of the 20th Annual Joint Conference of the IEEE Computer and Communications Societies, Apr. 22-26, IEEE Xplore Press, Anchorage, AK., pp: 1655-1663. DOI: 10.1109/INFCOM.2001.916662

HevinRajesh, D. and B. Paramasivan, 2012. Fuzzy based secure data aggregation technique in wireless sensor networks. J. Comput. Sci., 8: 899-907. DOI: 10.3844/jcssp.2012.899.907

Mota, L.T.M., 2005. Methods for load behavior prediction in power systems restoration. $\mathrm{PhD}$ Thesis, State University of Campinas (in portuguese).

Qian, Y. and C.N. Zhang, 2009. Secure broadcast in wireless sensor networks. Proceedings of the 3rd International Conference on Sensor Technologies and Applications, Jun. 18-23, IEEE Xplore Press, Athens, Glyfada, pp: 424-429. DOI: 10.1109/SENSORCOMM.2009.70

Yano, I.H., V.C.D. Oliveira, E.A.D.M. Fagotto, A.A. Mota and L.T.M. Mota, 2013. Predicting battery charge depletion in wireless sensor networks using received signal strength indicator. J. Comput. Sci., 9: 821-826. DOI: $10.3844 /$ jcssp.2013.821.826 